\title{
Exploiting Dimensions of the MIMO Wireless Channel: Multidimensional Link Adaptation
}

\author{
W. C. Freitas Jr., F. R. P. Cavalcanti and A. L. F. de Almeida \\ Teleinformatic Engineering Department \\ Federal University of Ceará (UFC) \\ Fortaleza-CE, Brazil \\ Emails: \{walter, rod, andre\}@gtel.ufc.br
}

\author{
R. R. Lopes \\ Communications Department \\ University of Campinas (Unicamp) \\ Campinas-SP, Brazil \\ Email: rlopes@decom.fee.unicamp.br
}

\begin{abstract}
In this paper, we propose an adaptive radio interface scheme for systems that employ multiple antennas. This is in contrast to the adaptive interface of single-antenna systems like in current packet data cellular systems. We propose groups of transmission parameters called Modulation, Coding and Antenna Schemes (MCAS) that are chosen according to the current conditions of the wireless channel, in order to maximize the performance in terms of goodput, while maintaining a certain level of reliability.
\end{abstract}

\section{INTRODUCTION}

Most of the wireless systems make its choice of the transmission parameters based on the worst case scenario. However, this strategy presents poor utilization of the resources when the system experiences good channel conditions. Therefore, a more clever strategy is to choose transmission parameters that depend on the current state of the wireless mobile channel, a process called link adaptation. For example, consider a Multiple-Input Multiple-Output (MIMO) system. If the MIMO channel is in a deep fade, then the system may choose a space-time code that provides a large diversity gain [1]. Otherwise, the system chooses a code with a high multiplexing gain. In other words, the system may exploit the diversity and multiplexing tradeoff available in MIMO systems to its advantage [2].

In this paper, we propose a link adaptation scheme that takes into account the presence of multiple antennas in both link ends. The idea is to dinamically adapt the signal transmission parameters (modulation level, code rate, antenna structure) to the current conditions of the wireless channel. We refer to the different sets of parameters as Modulation, Coding and Antenna Schemes (MCAS).

The remainder of this paper is organized as follows. Section II is dedicated to MIMO channel model as well as the system model. In section III, we present the MIMO architectures used in the proposed scheme. In section IV, we describe the multidimensional link adaptation strategy. Section V contains our simulation results and conclusions.

\section{SYSTEM AND CHANNEL MODELS}

Assume that the system has $M$ transmit and $N$ receive antennas. The transmitted signals are assumed to undergo independent fading, i.e., the signal at the output of each receive antenna is a superposition of quasi-static, slow and flat-faded versions of the transmitted signals, plus white Gaussian noise. For all the MIMO transmission schemes, we assume that the total transmission power is fixed (normalized to 1) and equally divided across the transmit antennas. Ideal symbol timing is assumed at the receiver. At discrete-time instant $k$, the received signal vector can be expressed as

$$
\mathbf{x}[k]=\mathbf{H s}[k]+\mathbf{n}[k],
$$

where $\mathbf{H}$ denotes the $N \times M$ MIMO channel matrix. The element $h_{n m}$ in $\mathbf{H}$ is the complex scalar channel that links the $m$ th transmit antenna and the $n$th receive antenna. The envelope of $h_{n m}$ follows a Rayleigh distribution. The $M \times 1$ vector $\mathbf{s}[k]$ contains the transmitted symbols (coded and modulated) at time-instant $k$. The $N \times 1$ vector $\mathbf{n}[k]$ denotes the temporally and spatially Additive White Gaussian Noise (AWGN).

\section{MIMO ARChIteCtURES}

Conventional MIMO transmission structures can provide either diversity gain or spatial multiplexing gain. The first gain is associated with link reliability, and results in a lower bit error rate (BER). The second one concerns the spectral efficiency of the overall system, i.e., the antennas are used to transmit as many bits as possible. Until the advent of hybrid MIMO schemes [3], MIMO schemes had to work in either one of these two classes, maximizing one of the gains while disregarding the other. However, it is well known that the focus in a particular gain implies a sacrifice of the other [2].

In general, the transmission process of a hybrid scheme can be divided in layers, where each layer is characterized by the type of gain it provides. Based on this concept of layers, hybrid MIMO transmission schemes combine pure diversity schemes (e.g. Space-Time Block Codes (STBC)) with pure spatial multiplexing schemes (e.g. Bell-Labs Layered Space Time (BLAST)). In hybrid systems, some layers are space-time coded across two, three or four antennas. For the remaining layers, a BLAST approach is used. With this idea, hybrid MIMO schemes achieve a compromise between spatial multiplexing and transmit diversity gains. In general, MIMO architectures can be classified in one of three groups depending of the provided gains: Pure Diversity Schemes, Pure 
Multiplexing Schemes and Hybrid MIMO Schemes. In the following, we briefly describe these MIMO architectures.

\section{A. Pure Diversity Schemes}

Space-Time Coding (STC) [1] is a well-known technique that provides diversity gain. Space-Time Codes use channel coding techniques combined with multiple transmit antennas, introducing temporal and spatial correlations into signals transmitted from different antennas, thus increasing the diversity order at the receiver. Two techniques widely used for STC are: Space-Time Block Codes (STBC) and Space-Time Trellis Codes (STTC). In the latter, when the number of antennas is fixed, the decoding complexity (measured by the number of trellis states at the decoder) increases exponentially as a function of the diversity level and transmission rate. In addressing the issue of decoding complexity, Alamouti [4] discovered a remarkable STBC scheme, denoted here as G2, for transmission with two antennas in flat fading channels. Due to its very simple structure, Alamouti's scheme is being considered for the Universal Mobile Telecommunications System (UMTS) standards. We now describe the G2 and other STBCs considered in this paper.

1) G2 STBC Scheme: in this scheme, two data symbols $s_{1}$ and $s_{2}$ are simultaneously transmitted by different antennas at a given symbol period $k$, where $s_{1}$ is the signal transmitted by antenna one and $s_{2}$ is the signal transmitted by antenna two. In the next symbol period $k+1$, antenna one transmits $-s_{2}^{*}$ and antenna two transmits $s_{1}^{*}$. The transmitted signals can be organized in the equivalent space-time coding matrix

$$
\boldsymbol{\Omega}_{G 2}[k, k+1]=\left[\begin{array}{cc}
s_{1} & -s_{2}^{*} \\
s_{2} & s_{1}^{*}
\end{array}\right],
$$

where the rows of $\boldsymbol{\Omega}_{G 2}[k, k+1]$ denote the transmit antennas, its columns denote the symbol period, and $(\cdot)^{*}$ means complex conjugation.

Due to the orthogonality of the transmit matrix $\Omega_{G 2}[k, k+$ 1], a simple linear operation in the receiver can be used to detect the transmit symbols $s_{1}$ and $s_{2}$, assuming that the channel is quasi-static and independent between two consecutive symbol periods, $[k]$ and $[k+1]$. Since the G2 scheme multiplexes $n_{s}=2$ useful symbols $\left(s_{1}\right.$ and $\left.s_{2}\right)$ in $n_{t}=2$ consecutive channel realizations, the effective spectral efficiency of this scheme is equal to $n_{s} / n_{t}=1 \cdot \log _{2} \mathcal{M}$ bps $/ \mathrm{Hz}$, where $\mathcal{M}$ is the cardinality of the modulation scheme considered. Schemes that achieves $n_{s} / n_{t}=1 \cdot \log _{2} \mathcal{M}$ bps $/ \mathrm{Hz}$ are also known as Full Rate (FR) schemes.

2) H3 STBC Scheme: in this scheme the transmitted signals can be organized in the equivalent space-time coding matrix

$$
\begin{aligned}
& \boldsymbol{\Omega}_{H 3}[k, k+1, k+2, k+3]= \\
& {\left[\begin{array}{cccc}
s_{1} & -s_{2}^{*} & \frac{s_{3}^{*}}{\sqrt{2}} & \frac{s_{3}^{*}}{\sqrt{2}} \\
s_{2} & s_{1}^{*} & \frac{s_{3}^{*}}{\sqrt{2}} & -\frac{s_{3}^{*}}{\sqrt{2}} \\
\frac{s_{3}}{\sqrt{2}} & \frac{s_{3}}{\sqrt{2}} & \frac{-s_{1}-s_{1}^{*}+s_{2}-s_{2}^{*}}{2} & \frac{s_{2}+s_{2}^{*}+s_{1}-s_{1}^{*}}{2}
\end{array}\right],}
\end{aligned}
$$

where the rows of $\boldsymbol{\Omega}_{H 3}[k, k+1, k+2, k+3]$ denote the transmit antennas, and its columns denote the symbol period. As with the scheme G2, a simple linear operation in the receiver can be used to detect the transmit symbols $s_{1}, s_{2}$ and $s_{3}$. However, in this case, the channels needs to be quasi-static and independent among four consecutive symbol periods, $[k],[k+1],[k+$ $2]$ and $[k+3]$. Since the H3 scheme multiplexes $n_{s}=3$ useful symbols $\left(s_{1}, s_{2}\right.$ and $\left.s_{3}\right)$ in $n_{t}=4$ consecutive channel realizations, the effective spectral efficiency of this scheme is equal to $n_{s} / n_{t}=(3 / 4) \cdot \log _{2} \mathcal{M} \mathrm{bps} / \mathrm{Hz}$.

\section{B. Pure Multiplexing Scheme}

Another point of view for multiple-antenna transmission is to focus on the maximization of the effective spectral efficiency. A well-known scheme proposed with this focus is the Bell Laboratories Layered Space-Time (BLAST) schemes (e.g. Vertically-BLAST and Diagonally-BLAST) [5]. In the V-BLAST scheme, all the antennas are used to multiplex different symbols in each symbol period. For instance, the transmitted signals at time instant $[k]$, considering three transmit antennas, can be organized the equivalent space-time coding matrix

$$
\boldsymbol{\Omega}_{V-B L A S T}[k]=\left[\begin{array}{l}
s_{1} \\
s_{2} \\
s_{3}
\end{array}\right],
$$

As spatially-multiplexed symbols cause Multiple Access Interference (MAI) in each other, signal processing is mandatory at the receiver in order to cancel MAI. In the following we describe both linear and non-linear approaches for MAI mitigation.

1) Linear Detection: in this paper, we consider only the MMSE algorithm for linear detection. At any time-instant $k$, the vector of output signal from the $M \times N$ MIMO-MMSE detector is given by

$$
\mathbf{y}[k]=\mathbf{W} \cdot \mathbf{x}[k],
$$

where

$$
\mathbf{W}=\left[\begin{array}{cccc}
w_{11} & w_{12} & \ldots & w_{1 N} \\
w_{21} & w_{22} & \ldots & w_{2 N} \\
\vdots & \vdots & \ddots & \vdots \\
w_{M 1} & w_{M 2} & \ldots & w_{M N}
\end{array}\right]
$$

We compute the error vector at the output of the MIMO-MMSE spatial filter as

$$
\mathbf{e}[k]=\mathbf{W} \mathbf{x}[k]-\mathbf{s}_{d}[k],
$$

where $\mathbf{s}_{d}[k]$ is the desired signal. Thus, the MMSE cost function can be expressed as

$$
J_{M M S E}=E\left\{\left\|\mathbf{W} \mathbf{x}[k]-\mathbf{s}_{d}[k]\right\|^{2}\right\} .
$$

The linear detector $\mathbf{W}$ that minimizes this cost funtion is given by [6]

$$
\mathbf{W}=\mathbf{R}_{\mathbf{s}_{d} \mathbf{x}} \cdot\left(\mathbf{R}_{\mathbf{x x}}\right)^{-1},
$$

where $\mathbf{R}_{\mathbf{x x}}=E\left\{\mathbf{x}[k] \mathbf{x}^{H}[k]\right\}$ and $\mathbf{R}_{\mathbf{s}_{d} \mathbf{x}}=E\left\{\mathbf{s}_{d}[k] \mathbf{x}^{H}[k]\right\}$ are the input covariance matrix and a cross-correlation matrix, respectively. 

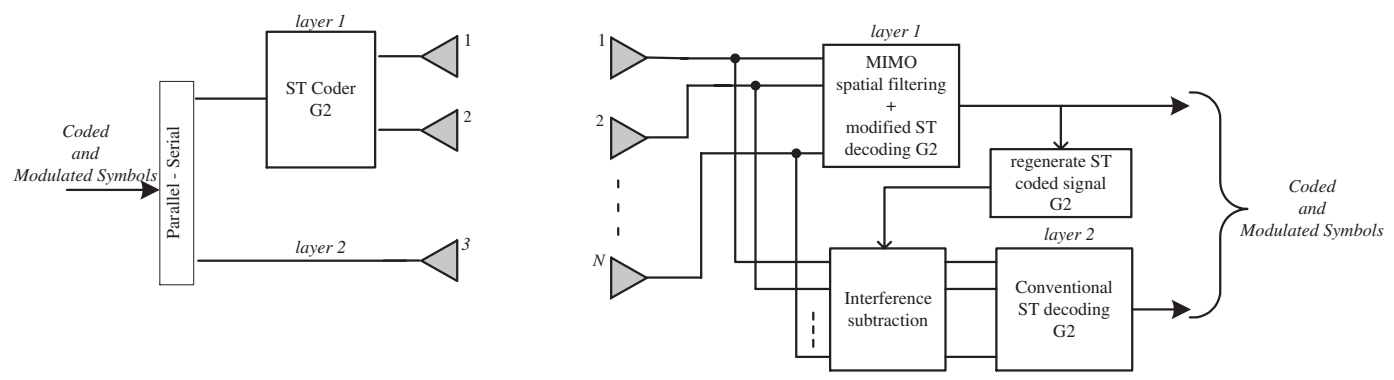

Fig. 1. Hybrid MIMO Structure (G2+1).

2) Non-Linear Detection: considering the linear approach just nulling of the interference layers is performed. A superior performance can be reached when a non-linear spatial-processing approach is used. In one such approach, the interference signal from layers that have already been detected are subtracted out of the received signal, in a decision-directed manner. Following this approach, we describe two algorithms below.

- Successive Interference Cancellation (SIC) - the layers are detected sequentially. Initially, the received signal $\mathbf{x}[k]$ goes through a linear detector for layer 1 , whose output is used to produce a hard estimate of the symbols at this layer, $\hat{z}_{1}[k]$. By doing so, the contribution of layer 1 to the received signal are estimated and cancelled, generating the signal $\mathbf{x}_{2}[k]$. In general, at the $i$-th layer, the signal $\mathbf{x}_{i}[k]$, hopefully free from the interference of layers $j<i$, is used for cancellation and decision. Then, based on the hard estimate of the symbols at this layer, $\hat{\mathbf{z}}_{i}[k]$, the contribution of this layer to the received signal is estimated and subtracted out from the "received signal" $\mathbf{x}_{i}[k]$. This procedure yields a modified received signal denoted by $\mathbf{x}_{i+1}[k]$ and given by

$$
\mathbf{x}_{i+1}[k]=\mathbf{x}_{i}[k]-\hat{z}_{i}[k] \mathbf{h}_{i}[k],
$$

where $\mathbf{h}_{i}$ is the $i$-th column of the matrix channel $\mathbf{H}$ corresponding to the channel gains associated to layer $i$, and $\hat{z}_{i}[k] \mathbf{h}_{i}[k]$ represents the estimated interference from the $i$-th layer. The result is that $\mathbf{x}_{i+1}$ is free from the interference coming from layers $1, \ldots, i$. This signal is then fed into the spatial filter for the $i+1$-th layer. This technique is also known as nulling and subtraction algorithm [7].

- Ordered Successive Interference Cancellation (OSIC) one of the disadvantages of SIC is that the signal associated with the first detection layer may exhibit a lower received SNR than that of the other layers. This may increase the probability of detection errors, which can propagate through the serial detection process, degrading performance of the overall receiver. This problem can be mitigated with an optimal ordering of the layers. When detection ordering is assumed, the first layer to be detected is that with the higher SNR. In this situation, the SIC approach turns into Ordered Successive Interference Cancellation (OSIC) [7].

\section{Hybrid MIMO Scheme}

In this section, we present the hybrid MIMO concept, a MIMO antenna scheme that makes simultaneous use of spatial multiplexing and transmit diversity. In a general way, the transmission process of a hybrid scheme can be divided into layers, where each layer is characterized by the provided gain: multiplexing or diversity. Although the hybrid approach is very general, in this paper we will use a 3-element transmit antenna array with two spatial multiplexing layers. Figure 1 shows the architecture of the G2+1 hybrid MIMO structure. A standard G2 (Alamouti's) [4] space-time block code is used at the first layer. The other layer is non-space-time-coded, and operates in a co-channel way with the G2 layer, following the V-BLAST idea. In the $\mathrm{G} 2+1$ scheme, the transmitted signals can be organized in the equivalent space-time coding matrix

$$
\boldsymbol{\Omega}_{G 2+1}[k, k+1]=\left[\begin{array}{cc}
s_{1} & -s_{2}^{*} \\
s_{2} & s_{1}^{*} \\
s_{3} & s_{4}
\end{array}\right] .
$$

From (11), it can be seen that $n_{s}=4$ useful symbols (two from each multiplexing layer) are transmitted in $n_{t}=$ 2 consecutive channel realizations $([k, k+1])$. Thus, the effective spectral efficiency of this scheme is equal to $n_{s} / n_{t}=$ $2 \cdot \log _{2} \mathcal{M}$ bps/Hz. Compared to the G3 STC [1], this hybrid scheme offers a $300 \%$ increase in data rate, since the G3 schemes just achieve the effective spectral efficiency of $(1 / 2)$. $\log _{2} \mathcal{M}$ bps/Hz.

1) Modified MAI algorithm for the Hybrid MIMO Scheme: in the case of the Hybrid MIMO scheme (G2+1) we have two layers: a standard G2 (Alamouti's) space-time block code at the first layer and a non-space-time-coded layer. Thus, we can adapt the MAI cancellation algorithm in such a way that the orthogonal structure of the space-time code (G2) is preserved as much as possible in its output signal.

In this case, we obtain the error vector at the output of the MIMO-MMSE spatial filter as

$$
\mathbf{e}[k]=\mathbf{W} \mathbf{x}[k]-\mathbf{H}_{d} \mathbf{s}_{1}[k]=\mathbf{W} \mathbf{x}[k]-\mathbf{x}_{d}[k],
$$

where $\mathbf{x}_{d}[k]=\mathbf{H}_{d} \mathbf{s}_{1}[k]$ is the desired space-time coded signal associated to the first multiplexing layer of a particular 
TABLE I

Modulation, Coding ANd Antenna Schemes.

\begin{tabular}{|c|c|c|c|c|}
\hline MCAS & $\begin{array}{c}\text { Rate } \\
\text { Code }\end{array}$ & $\begin{array}{c}\text { Antenna } \\
\text { Schemes }\end{array}$ & $\begin{array}{c}\text { Useful } \\
\text { Payload } \\
\left.\text { (Bits/ } / T_{f}\right)\end{array}$ & $\begin{array}{c}\text { Max Normalized } \\
\text { Goodput }-G P_{\max } \\
\left(\text { Bits } / T_{s}\right)\end{array}$ \\
\hline 1(QPSK) & $1 / 2$ & $\mathrm{H} 3$ & 106 & 0.7361 \\
\hline $2(\mathrm{QPSK})$ & $1 / 2$ & $\mathrm{G} 2+1$ & 284 & 1.972 \\
\hline $3(\mathrm{BPSK})$ & 1 & V-BLAST & 424 & 2.944 \\
\hline
\end{tabular}

hybrid transmission scheme. Contrarily to the classical MIMO-MMSE spatial filter, where the desired signal is $\mathbf{s}_{d}[k]$, here the desired signal consists of the original transmitted signal modified by desired MIMO channel response $\mathbf{H}_{d}$, which can be interpreted as the virtual" channel between the transmitter and the output of the spatial filter.

The MMSE cost function may be written as

$$
J_{M M S E}=E\left\{\left\|\mathbf{W} \mathbf{x}[k]-\mathbf{x}_{d}[k]\right\|^{2}\right\} .
$$

The optimal coefficients are found by minimizing the above cost function with respect to $\mathbf{W}$. The solution is given by

$$
\mathbf{W}=\mathbf{R}_{\mathbf{x}_{d} \mathbf{x}} \mathbf{R}_{\mathbf{x x}}{ }^{-1},
$$

where $\mathbf{R}_{\mathbf{x x}}=E\left\{\mathbf{x}[k] \mathbf{x}^{H}[k]\right\}$ and $\mathbf{R}_{\mathbf{x}_{d} \mathbf{x}}=E\left\{\mathbf{x}_{d}[k] \mathbf{x}^{H}[k]\right\}$ are the input covariance matrix and a cross-correlation matrix, respectively.

\section{Multidimensional Link Adaptation}

In this section we present a multidimensional link adaptation scheme with three transmission modes. We use a rate-1/2 convolutional code as channel encoder, with generator polynomial represented in octal form as $(171,133)$. We choose BPSK and QPSK as modulation schemes. We assume a system with three transmit and $N$ receive antennas (3Tx-NRx).

We considered two classical MIMO schemes, Tarokh's (H3) STBC [1] and Foschini's Vertical BLAST (V-BLAST) scheme [5]. We also consider the Hybrid MIMO Structure (G2+1) [3] described in the previous section, which combines the advantages of the two classical schemes.

Table I summarizes our choice for the MCAS parameters. In each MCAS, we assume the transmission of blocks (frames) of 144 payload symbols. We used an 8-bit Cyclic Redundancy Check (CRC) scheme for block error detection. Thus, if the convolutional code fails in its task of error correction, this failure is detected by the CRC. In this case, the whole block is assumed to be in error. The Block Error Rate (BLER) measures the probability of this event.

With the BLER performance, shown in Figs. 2 and 3, for each MCAS we can calculate the Normalized Goodput $(G P)$, using

$$
G P=G P_{\max } \cdot(1-\mathrm{BLER}) .
$$

For each MCAS, the Useful Payload is defined as the number of information bits delivered in a frame period $\left(T_{f}\right)$. Let

$$
T_{s}=T_{f} / 144
$$

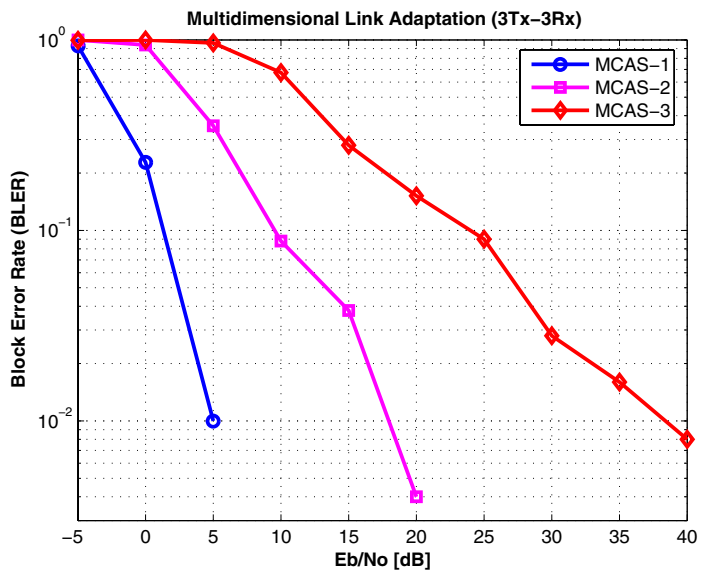

Fig. 2. BLER performance considering 3 transmit and receive antennas.

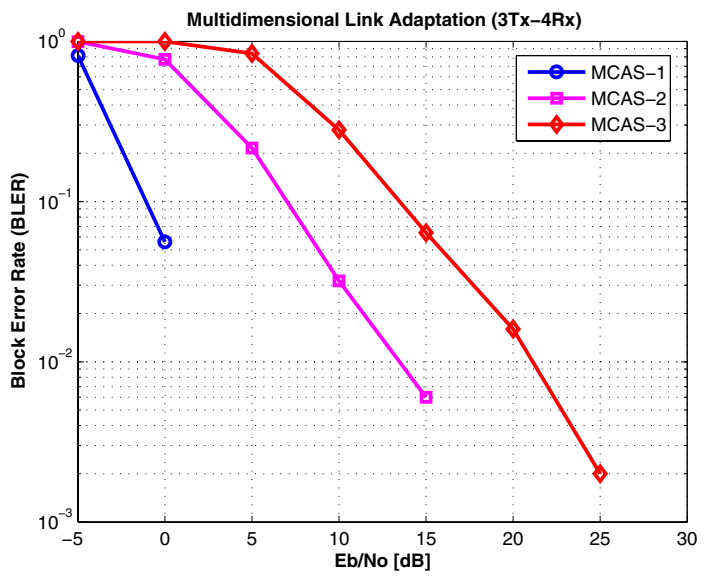

Fig. 3. BLER performance considering 3 transmit and 4 receive antennas.

be the symbol period. Still in Table I, the Max Normalized Goodput $\left(G P_{\max }\right)$ is defined as the total number of information bits divided by the symbol period. Assuming that bits received in error are ignored, and thus count as overhead bits, this is the maximum rate achievable by the system, since it assumes that all bits are correctly received.

\section{Results AND CONCLUSIONS}

In this section, we show the BLER performance vs. Eb/No and Normalized Goodput $(G P)$ performance vs. Eb/No in (dB) for the proposed MCAS by means of Monte Carlo simulations, considering three and four receive antennas. For the hybrid scheme we consider as MAI cancellation algorithm the OSIC strategy.

In Figs. 4 and 5 we show the normalized goodput performance of the proposed multidimensional link adaptation scheme. We assume that 500 frames consisting of 144 symbols are transmitted. Fig. 4 shows the result for three receive antennas. Clearly, to maximize the goodput, for low $\mathrm{Eb} / \mathrm{No}$ we should use the most robust MCAS (MCAS-1) and 


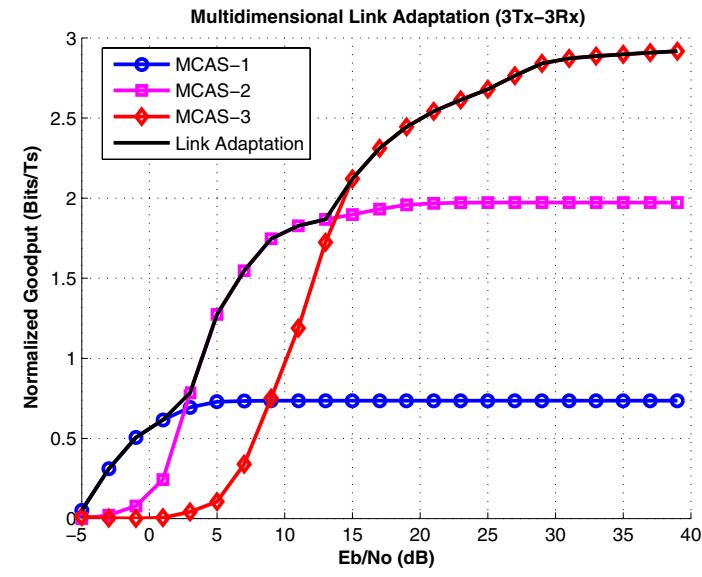

Fig. 4. MCAS Goodput considering 3 transmit and receive antennas.

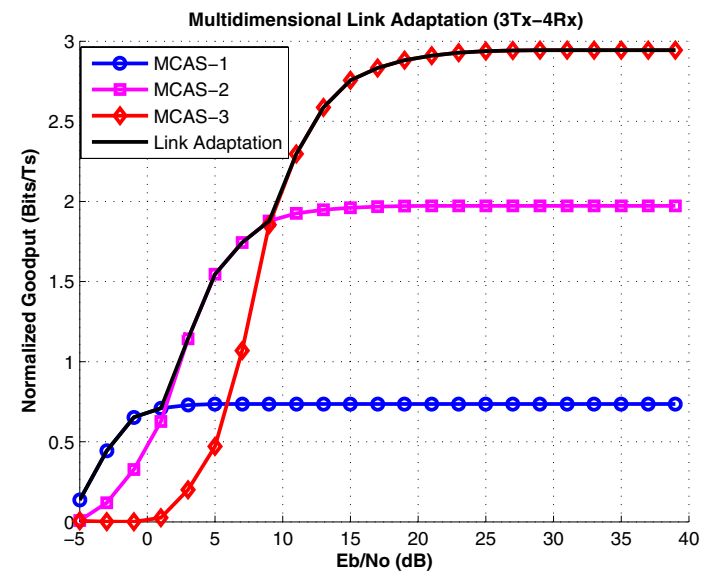

Fig. 5. MCAS Goodput considering 3 transmit and 4 receive antennas.

switch to other MCAS (MCAS-2 and MCAS-3) when these present a higher performance in terms of normalized goodput. This is because, when channel conditions improve, they can multiplex almost two and three bits in a single symbol period, respectively. This result corroborates the idea that hybrid receivers offer a compromise between a pure diversity scheme (H3) and a pure multiplexing scheme (V-BLAST).

In Fig. 5, we evaluate the system performance when four receive antennas are used. Clearly, we can see that using one more antenna greatly improves the performance of MCAS-3. For example, at $10 \mathrm{~dB}$ of Eb/No MCAS-3 has a normalized goodput about $1 \mathrm{Bits} / \mathrm{Ts}$ for three receive antennas, and 2.2 Bits/Ts for four receive antennas. Regarding the performance of the MCAS- 1 and MCAS- 2 with four receive antennas, we see in Fig. 5 that the normalized goodput performance of the MCAS-2 is closer than the performance of the MCAS-1. This can be explained by the higher diversity order of the second layer of the hybrid G2+1 scheme, considering the OSIC as MAI algorithm.

Finally, Figs. 4 and 5 indicate that the transmission parameters should be changed at each crossing of the MCAS curves, thus maximizing the normalized goodput for all values of $\mathrm{Eb} / \mathrm{No}$. Hence, the receiver can measure the received $\mathrm{Eb} / \mathrm{No}$ and report to the transmitter which MCAS should be used. Since our scheme of multidimensional link adaptation involves only three schemes, only two bits are necessary to tell the transmitter that a switch to a given scheme is needed, which represents a low overhead.

\section{ACKNOWLEDGMENT}

The authors are with the Wireless Telecom Research Group (GTEL) at the Federal University of Ceará, Fortaleza-CE, Brazil. W. C. Freitas Jr. is supported by FUNCAP, A. L. F. de Almeida is supported by CAPES and R. R. Lopes is supported by FAPESP, grant no. 03/07814-1.This work was supported by a grant from Ericsson of Brazil Research Branch under ERBB/UFC.11 Technical Cooperation Contract (URL: http://www.ericsson.ufc.br). Also, the authors would like to thank Charles C. Cavalcante, the coordinator of ERBB/UFC.11.

\section{REFERENCES}

[1] V. Tarokh, H. Jafarkhani, and A. R. Calderbank, "Space-time block codes from orthogonal designs," IEEE Transactions on Information Theory, vol. 5, pp. 1456-1467, Jul 1999.

[2] L. Zheng and D. Tse, "Diversity and multiplexing: a fundamental tradeoff in multiple antenna channels," in IEEE Transactions on Information Theory, vol. 49, May 2003, pp. 1073-96.

[3] A. L. F. de Almeida, W. C. Freitas Jr., F. R. P. Cavalcanti, J. C. M. Mota, and R. L. de Lacerda Neto, "Performance of a MIMO systems with a hybrid of transmit diversity and spatial multiplexing," XX Simpósio Brasileiro de Telecomunicações (SBrT), 2003, Rio de Janeiro-RJ, Brasil.

[4] S. Alamouti, "A Simple Transmit Diversity Technique for Wireless Communications," IEEE Journal of Selected Areas in Communications, vol. 16, pp. 1451-1458, Oct 1998.

[5] G. J. Foschini and M. J. Gans, "On limits of wireless communications in a fading environment when using multiple antennas", Wireless Personal Communications, v. 6, n. 3, Mar 1998, pp. 311-335.

[6] S. Haykin, Adaptive Filter Theory, Prentice-Hall, 1996.

[7] Branka Vucetic and Jinhong Yuan, Space-Time Coding, Wiley, 2003. 